\title{
BIODIESEL METÍLICO DE Dipteryx lacunifera: PREPARAÇÃO, CARACTERIZAÇÃo E EFEITO DE ANTIOXIDANTES NA ESTABILIDADE À OXIDAÇÃO
}

\author{
Francisca Diana da Silva Araújo, Carla Verônica Rodarte de Moura e Mariana H. Chaves* \\ Departamento de Química, Universidade Federal do Piauí, Campus Ministro Petrônio Portela, 64049-550 Teresina - PI, Brasil
}

Recebido em 1/11/09; aceito em 22/5/10; publicado na web em 1/9/10

\begin{abstract}
METHYL BIODIESEL FROM Dipteryx lacunifera: PREPARATION, CHARACTERIZATION AND EFFECT OF ANTIOXIDANT ON THE OXIDATION STABILITY. The objective of this work was to study the potentialities of Dipteryx lacunifera Ducke seeds oil for biodiesel production. The yield in oil was of $46.11 \pm 0.37 \%$. Methyl biodiesel was prepared by base catalyzed transesterification yielding $88.13 \%$ and percentage conversion of triglycerides to corresponding methyl ester of $89.1 \%$. The addition of the tertiary butylhydroquinone (TBHQ, 50 ppm), butylated hydroxytoluene (BHT, 50 ppm) and cashew nut shell liquid (CNSL, 2000 ppm) antioxidants in the biodiesel contributed to the increase of the induction period of $2.97 \mathrm{~h}$ for $8.08 ; 6.06$ and $6.02 \mathrm{~h}$, respectively.
\end{abstract}

Keywords: Dipteryx lacunifera; biofuel; antioxidant.

\section{INTRODUÇÃO}

As pesquisas com biodiesel se intensificaram nos últimos anos, por se tratar de um biocombustível para uso em motores a combustão interna, capaz de substituir parcial ou totalmente o petrodiesel, porém com vantagens ambientais, pois é livre de enxofre e de compostos aromáticos, emite menor índice de particulados, não é tóxico, é biodegradável, oriundo de fontes renováveis e, como consequência, o mundo se beneficiará com a menor emissão de gases que contribuem para o aumento do efeito estufa. ${ }^{1,2}$

Quimicamente, o biodiesel é uma mistura de ésteres alquílicos de ácidos graxos, derivados de óleos vegetais ou de gorduras animais, podendo ser obtido por vários métodos, dentre os quais, a transesterificação dos triacilglicerois com alcoóis de cadeia curta, como o metanol ou etanol, em presença de um catalisador homogêneo básico, ainda é o processo industrialmente mais viável. ${ }^{2-4}$

O Brasil apresenta um elevado potencial para a produção de biodiesel, devido à sua grande extensão territorial com áreas produtivas e a existência de diversas oleaginosas como opções de matérias-primas, proporcionada pela variedade de clima e solo. ${ }^{5}$ Devido à biodiversidade de oleaginosas no país, ainda existem plantas nativas que são pouco conhecidas ou divulgadas, tornando-se imprescindível a realização de estudos para se avaliar a capacidade de produção destas espécies de acordo com a vocação regional para produção e consumo de combustíveis da biomassa.

Dipteryx lacunifera Ducke, sinonímia Coumarouna lacunifera Ducke, pertence à família Leguminoseae-Papilionoideae e é uma planta nativa da América do Sul. No Brasil, é encontrada no Maranhão e Piauí, onde é conhecida popularmente como garampara, castanha-de-burro e castanha-do-gurguéia. ${ }^{6}$ As amêndoas desta espécie são ricas em óleo (39,25-45,6\%), com composição química que inclui os ácidos: cáprico (C10:0; 0,0-0,8\%), láurico (C12:0; 0,0-1,2\%), palmítico (C16;0; 10,4$15,9 \%)$, linolênico (C8:3; 0,0-3,0\%), linoleico (C18:2; 1,76-14,4\%), oleico $(\mathrm{C} 18: 1 ; 36,1-75,8 \%)$, esteárico $(\mathrm{C} 18 ; 0 ; 4,6-7,3 \%)$, araquidônico (C20:4; 0,0-8,7\%), araquídico (C20:0; 0,0-3,4\%), erúcico (C22:1; 0,01,4\%), behênico (C22:0; 0,0-4,2\%) e lignocérico (C24:0; 0,6-3,5\%).7.8

Considerando a inexistência de estudos explorando as propriedades agroenergéticas da $D$. lacunifera, o presente trabalho teve como objetivos estudar as potencialidades do óleo das amêndoas

*e-mail: mariana@ufpi.edu.br desta espécie para a produção de biodiesel e investigar o efeito de antioxidantes no aumento da estabilidade à oxidação do biodiesel.

\section{PARTE EXPERIMENTAL}

\section{Instrumentação}

A viscosidade cinemática foi determinada utilizando-se viscosímetro Quimis ${ }^{\circledR}$, de acordo com a norma ASTM D 445. A determinação da estabilidade à oxidação foi feita no Rancimat Metrohm 743, conforme norma EN 14112; a medida do ponto de fulgor foi em aparelho de vaso fechado Pensky Martens APM-7 (ASTM D 93) e a corrosividade ao cobre em equipamento Petrotest ${ }^{\circledR}$ (ASTM D 130).

Os percentuais de glicerina livre e total, mono-, di- e triacilglicerois foram obtidos em um cromatógrafo gasoso com detector de ionização de chama (CG-DIC), Varian 3800 e coluna DB-5. O teor de metanol foi determinado em CG-DIC acoplado a headspace estático, Varian 3900 e coluna DB-1.

Os ésteres metílicos foram analisados em CG-EM da Shimadzu, modelo GC17A e detector de massas QP5000, utilizando coluna DB-5. As análises de RMN foram obtidas em espectrômetro Varian Inova 500 (500 MHz); as de infravermelho em um Nicolet, Impact 400 e as termogravimétricas em balança termogravimétrica TGA2050 da TA Instruments.

\section{Coleta do material vegetal}

Os frutos de garampara foram fornecidos pelo Dr. E. C. E. Araújo da Embrapa Meio-Norte, Teresina-PI, sendo coletados no município de Bom Jesus, Piauí, em setembro de 2005 e acondicionados em sacos de papel à temperatura de aproximadamente $25^{\circ} \mathrm{C}$, por um período de 1 ano. A identificação botânica foi feita pelo Dr. H. C. de Lima do Instituto de Pesquisa Jardim Botânico do Rio de Janeiro e exsicatas da espécie foram depositadas nos Herbários Graziela Barroso - UFPI e da Embrapa Meio-Norte sob os números TEPB 18.246 e 1704, respectivamente.

\section{Extração e degomagem do óleo}

Após a retirada das cascas dos frutos, amostras de aproximadamente $10 \mathrm{~g}$ de amêndoas foram trituradas, desumidificadas em estufa 
a $105^{\circ} \mathrm{C}$ e submetidas ao processo de extração contínua do óleo com o uso de hexano, em aparelhagem Soxhlet por $6 \mathrm{~h} .{ }^{9} \mathrm{O}$ rendimento do óleo foi obtido pela expressão: Rendimento $(\%)=$ (massa do óleo/ massa da amostra) x 100 .

Para obtenção do biodiesel, cerca de $500 \mathrm{~mL}$ de óleo, extraído nas mesmas condições anteriores, foi previamente degomado com $\mathrm{H}_{3} \mathrm{PO}_{4}$, de acordo com Moretto. ${ }^{10}$

\section{Determinação da composição em ácidos graxos no óleo}

Os triacilglicerois foram transesterificados, seguindo metodologia IUPAC, ${ }^{11}$ com algumas modificações, através do seguinte procedimento: em tubo de ensaio, pesou-se cerca de $100 \mathrm{mg}$ do óleo, adicionou-se $2 \mathrm{~mL}$ de hexano e $0,2 \mathrm{~mL}$ de solução metanólica $2 \mathrm{~mol} \mathrm{~L}^{-1}$ de KOH. Agitou-se o frasco por $20 \mathrm{~min}$ em agitador vortex e adicionou-se $2 \mathrm{~mL}$ de solução saturada de cloreto de sódio, até a separação da fase orgânica. ${ }^{12,13}$ Os ésteres metílicos dos ácidos graxos foram analisados através de injeção de $1 \mu \mathrm{L}$ da fase orgânica em cromatógrafo gasoso acoplado a espectrômetro de massas (CGEM), utilizando coluna de $30 \mathrm{~m}$ de comprimento, com $0,25 \mathrm{~mm}$ de diâmetro interno e $0,25 \mu \mathrm{m}$ de espessura do filme. As condições de operação foram: temperatura programada da coluna de $150{ }^{\circ} \mathrm{C}$ por $2 \mathrm{~min}$, taxa de aquecimento $5{ }^{\circ} \mathrm{C} \mathrm{min}{ }^{-1}$ até $230{ }^{\circ} \mathrm{C}$ por 7 min e $4{ }^{\circ} \mathrm{C}$ $\mathrm{min}^{-1}$ até $260{ }^{\circ} \mathrm{C}$ mantida por $10 \mathrm{~min}$; temperatura do injetor: 230 ${ }^{\circ} \mathrm{C}$; temperatura da interface: $250{ }^{\circ} \mathrm{C}$; gás de arraste: hélio, com fluxo de $0,6 \mathrm{~mL} \mathrm{~min}^{-1}$. A corrida com detector de massa foi feita na forma scan, com tempo de aquisição de 42,5 min e corte do solvente em 4 min; faixa de massa: 40 a 400 Daltons; voltagem do filamento: $70 \mathrm{eV}$; voltagem do detector; $1,3 \mathrm{KV}$. A identificação dos ésteres metílicos foi feita através de comparação dos espectros de massas obtidos com os da biblioteca Wiley229.

\section{Obtenção do biodiesel e análise por CCD e RMN ${ }^{1} \mathbf{H}$}

O óleo degomado com ácido fosfórico $(400 \mathrm{~mL})$ foi convertido em biodiesel por meio de reação de transesterificação alcalina. Hidróxido de sódio foi dissolvido em metanol e adicionado ao óleo, nas razões mássicas de 100:20:1 (óleo: $\mathrm{MeOH}: \mathrm{NaOH}$ ), que corresponde aproximadamente à razão molar de 1:6 (óleo:MeOH) com $1 \%$ de catalisador. ${ }^{1} \mathrm{~A}$ mistura foi mantida sob agitação à temperatura ambiente por $1 \mathrm{~h},{ }^{14,15}$ posteriormente, foi transferida para funil de decantação e os ésteres metílicos foram separados da glicerina, lavados com água e desumidificados a $100^{\circ} \mathrm{C}$. O biodiesel obtido foi analisado por cromatografia em camada delgada (CCD) de gel de sílica, usando como eluente hexano-AcOEt (95:5) e vapores de iodo como revelador. O rendimento da reação foi calculado pela expressão: Rendimento (\%) $=$ (massa do biodiesel $/$ massa do óleo $) \times 100$.

A taxa de conversão dos triacilglicerois do óleo em biodiesel metílico foi determinada pela análise do espectro de $\mathrm{RMN}{ }^{1} \mathrm{H}$ obtido a partir do biodiesel dissolvido em $\mathrm{CDCl}_{3}$ e usando a seguinte expressão: $C=100 \times 2 A_{\mathrm{CH}_{3}} / 3 A_{\mathrm{CH}_{2}}$, onde $A_{\mathrm{CH}_{3}}$ é a integração do sinal em $\delta$ 3,7 dos hidrogênios metoxílicos e $A_{\mathrm{CH}_{2}}$ é a integração do sinal em $\delta$ 2,3 dos hidrogênios metilênicos $\alpha$-carbonílico da molécula de éster. ${ }^{16}$

\section{Caracterização físico-química, análises na região do infravermelho e termogravimétrica do óleo e do biodiesel}

As análises físico-químicas do biodiesel foram realizadas conforme as normas da American Society of Testing and Materials (ASTM) e Comité Européen de Normalisation (CEN) indicadas pela Resolução ${ }^{\circ}$ 07/2008 da Agência Nacional de Petróleo, Gás Natural e Biocombustíveis (ANP). ${ }^{3} \mathrm{O}$ índice de acidez do óleo e do biodiesel e o índice de saponificação do óleo foram determinados de acordo com as Normas Analíticas do Instituto Adolfo Lutz. ${ }^{9}$

Os espectros de infravermelho do óleo degomado e do biodiesel foram obtidos na região de 4000-400 $\mathrm{cm}^{-1}$, em partilhas de $\mathrm{KBr}$.

As curvas TG do óleo degomado e do biodiesel de garampara foram obtidas em um intervalo de temperatura de 30 a $600{ }^{\circ} \mathrm{C}$, com uma razão de aquecimento de $10{ }^{\circ} \mathrm{C} \mathrm{min}^{-1}$, em atmosfera de nitrogênio com vazão de $50 \mathrm{~mL} \mathrm{~min}^{-1}$, utilizando uma panela de alumínio com orifício na tampa de aproximadamente $0,5 \mathrm{~mm}$ de diâmetro. A perda de massa da amostra foi determinada como a diferença entre a massa inicial e final. O ponto de ebulição foi considerado como o ponto de intersecção da tangente da inclinação de perda de massa com a linha de base inicial (temperatura onset). ${ }^{17-19} \mathrm{O}$ software do equipamento foi usado para desenhar as linhas tangentes e computar a temperatura onset.

\section{Testes de estabilidade à oxidação}

A estabilidade à oxidação foi medida segundo a norma EN 14112, utilizando amostras de $3 \mathrm{~g}$ de biodiesel, as quais foram analisadas sob aquecimento com temperatura de $110^{\circ} \mathrm{C}$ e fluxo de ar constante de $10 \mathrm{~L} \mathrm{~h}^{-1}$. No Rancimat, o fluxo de ar passa através da amostra, sendo borbulhado posteriormente em um frasco contendo água deionizada ou destilada. O fluxo de ar arrasta os ácidos carboxílicos voláteis (produtos de oxidação), que se solubilizam e aumentam a condutividade da água. A resposta obtida é uma curva de condutividade elétrica versus o tempo, na qual se constroem duas tangentes que se interceptam num ponto, correspondente, na escala do tempo, ao período de indução ou estabilidade à oxidação. ${ }^{20}$

O biodiesel metílico de garampara foi submetido ao tratamento com dois antioxidantes sintéticos, TBHQ (terc-butil-hidroquinona) e BHT (butil-hidroxi-tolueno), ambos da Sigma, USA, e um de origem natural, o LCC (líquido da casca da castanha de caju), obtido por extração com hexano, a partir das cascas da castanha em aparelhagem Soxhlet.

\section{Análise estatística}

À exceção de alguns parâmetros físico-químicos, as demais análises foram realizadas em triplicata, sendo apresentados a média e o desvio padrão das medidas.

\section{RESULTADOS E DISCUSSÃO}

O rendimento obtido para o óleo de garampara foi de $46,1 \pm$ $0,4 \%$, aproximadamente igual ao maior teor relatado na literatura (39,3-45,6\%). ${ }^{7,8}$ Esse valor é relativamente elevado ao se comparar com os óleos de soja (18\%), algodão (15\%) e amendoim (40-43\%), encontrando-se na faixa observada para a mamona (45-50\%), colza $(40-48 \%)$ e girassol $(38-48 \%) .^{21}$

O óleo de garampara degomado apresentou índice de acidez igual a $0,17 \pm 0,01 \mathrm{mg}$ de $\mathrm{KOH} \mathrm{g}{ }^{-1}$ de óleo, portanto, dentro dos limites observados para óleos refinados (inferior a $6 \mathrm{mg} \mathrm{KOH} \mathrm{g}^{-1}$ de óleo) ${ }^{12}$ e com um valor adequado para o preparo do biodiesel (menor do que $1 \mathrm{mg} \mathrm{KOH} \mathrm{g}^{-1}$ de óleo). ${ }^{16}$

$\mathrm{O}$ índice de saponificação do óleo de garampara mostrou-se inferior ao do pinhão-manso (Jatropha curcas) (192 $\mathrm{mg} \mathrm{KOH} \mathrm{g}^{-1} \mathrm{de}$ óleo), cuja composição química é predominantemente oleica (C18:1) e linoleica (C18:2), ${ }^{22}$ sugerindo a presença de triacilglicerois de ácidos graxos com cadeia hidrocarbônica mais longa no óleo de garampara.

O óleo apresentou o ácido oleico (C18:1; 64,71 \1,35\%) como componente majoritário dos triacilglicerois e com percentual dentro do intervalo relatado na literatura $(36,10-75,82 \%) \cdot{ }^{7,8}$ Os ácidos graxos insaturados e saturados somaram 70,66 e $30,78 \%$, respectivamente. 
Verificou-se ainda a ocorrência de quantidades significativas dos ácidos C20, C22 e C24 (Tabela 1), comprovando o que foi sugerido pelo valor do índice de saponificação determinado.

Tabela 1. Composição em ácidos graxos do óleo das amêndoas de garampara (\% $\mathrm{m} / \mathrm{m}$ de ésteres metílicos)

\begin{tabular}{lc}
\hline Ácido graxo & $\% \pm \mathrm{DP}$ \\
\hline Palmítico, C16:0 & $8,55 \pm 2,79$ \\
Linoleico (C18:2 Z,Z 9,12) & $1,59 \pm 0,20$ \\
Oleico (C18:1 Z 9) & $64,71 \pm 1,35$ \\
Esteárico, C18:0 & $3,72 \pm 0,61$ \\
Gadoleico, C20:1 Z 9 & $3,54 \pm 0,21$ \\
Araquídico C20:0 & $1,23 \pm 0,18$ \\
Erúcico, C22:1 Z 9 & $0,82 \pm 0,23$ \\
Behênico, C22:0 & $10,42 \pm 2,84$ \\
Lignocérico, C24:0 & $6,86 \pm 2,37$ \\
Ácidos graxos insaturados & 70,66 \\
Ácido graxos saturados & 30,78 \\
Total identificado & 100 \\
\hline
\end{tabular}

DP: desvio padrão

\section{Análise do biodiesel de garampara}

Biodiesel metílico do óleo de garampara foi preparado por transesterificação em meio alcalino, sendo seu processo de separação da fase glicerinada de fácil execução, não apresentando a formação de emulsão.

A análise por CCD sugeriu a ocorrência da reação de transesterificação por apresentar, para o biodiesel, uma mancha com fator de retenção maior do que o observado para os triacilglicerois do óleo. $\mathrm{O}$ espectro de $\mathrm{RMN}{ }^{1} \mathrm{H}$ confirmou a formação do produto transesterificado, exibindo sinal de metoxila em $\delta 3,30$, característico de ésteres metílicos..$^{23} \mathrm{O}$ biodiesel metílico foi obtido com rendimento satisfatório de $88,1 \%$ e conversão em ésteres de $89,1 \%$, determinado por $\mathrm{RMN}{ }^{1} \mathrm{H}^{1,23}$

As principais absorções observadas no espectro de IV (Figura $1 \mathrm{~S}$, material suplementar) do óleo de garampara foram em $1745 \mathrm{~cm}^{-1}$ (vibração de estiramento $\mathrm{C}=\mathrm{O}$ ), 1234, 1163 e $1105 \mathrm{~cm}^{-1}$ (estiramento C-O) referentes aos triacilglicerois. Estas absorções foram também observadas no espectro do biodiesel com pequenas variações: 1743 $\mathrm{cm}^{-1}(\mathrm{C}=\mathrm{O}), 1244,1174$ e $1097 \mathrm{~cm}^{-1}(\mathrm{C}-\mathrm{O})$. Adicionalmente, o espectro de IV do biodiesel apresentou uma absorção fraca na região de $3500 \mathrm{~cm}^{-1}$, característico de estiramento $\mathrm{OH}$, atribuído à presença de resíduos de metanol, conforme evidenciado na análise por CG.

\section{Análise termogravimétrica do óleo e biodiesel}

Na análise TG/DTG do óleo degomado (Figura 1) foi observado um primeiro evento térmico com perda de massa de $89,13 \%$, no intervalo de temperatura de $251,94-445,68^{\circ} \mathrm{C}\left(T_{\text {ouset }} 381,84^{\circ} \mathrm{C}\right)$, atribuído à volatilização dos triacilglicerois. Um segundo evento térmico, com perda de massa igual a 8,65\%, ocorreu no intervalo de 445,68-492,60 ${ }^{\circ} \mathrm{C}\left(T_{\text {onset }} 463,00{ }^{\circ} \mathrm{C}\right)$, possivelmente promovido por triacilglicerois de cadeia mais longa. ${ }^{1}$ Verificou-se ainda uma pequena perda de massa de $0,73 \%$ entre $86,96-124,00{ }^{\circ} \mathrm{C}$, provavelmente atribuída à perda de umidade ou de alguma substância volátil presente no óleo.

A curva TG/DTG do biodiesel metílico de garampara (Figura 1) exibiu três eventos térmicos. Os dois eventos principais ocorreram nos intervalos de $64,26-281,46{ }^{\circ} \mathrm{C}\left(T_{\text {onset }} 158,32{ }^{\circ} \mathrm{C}\right)$
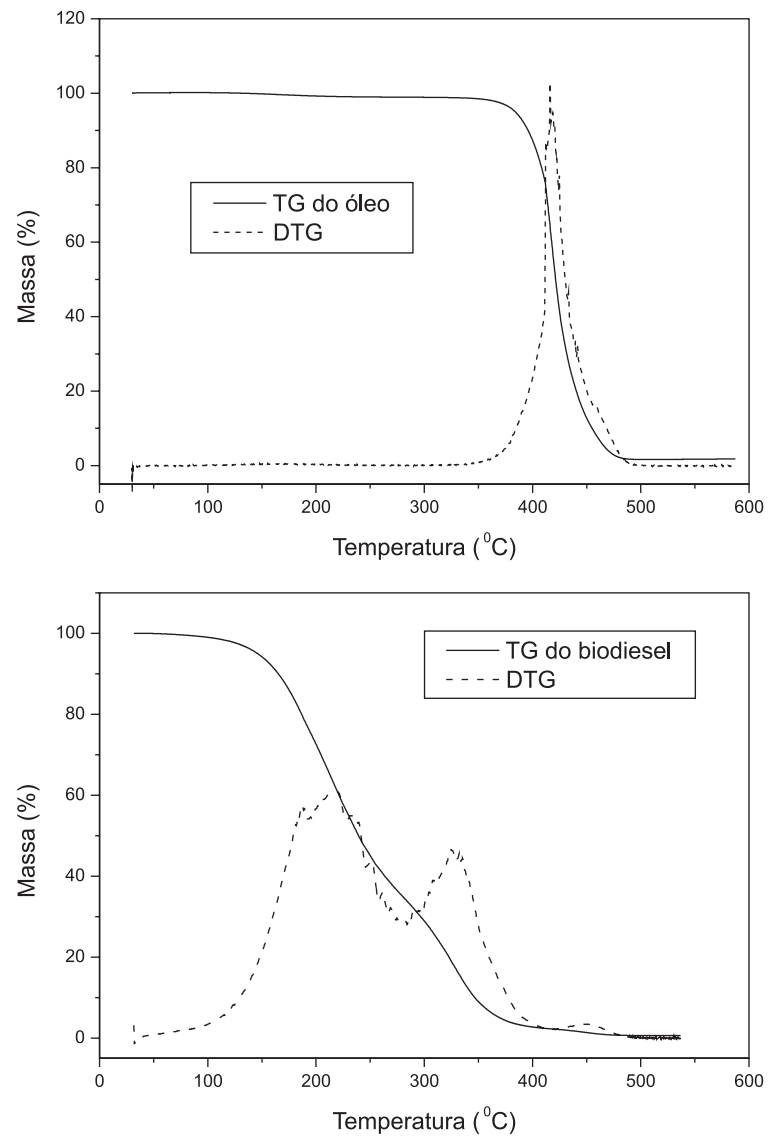

Figura 1. Curvas TG/DTG do óleo degomado e biodiesel metílico de garampara

e $281,46-418,44{ }^{\circ} \mathrm{C}\left(T_{\text {onset }} 358,23{ }^{\circ} \mathrm{C}\right)$ com perdas de massa de 65,16 e $32,30 \%$, respectivamente, os quais foram associados à volatilização dos ésteres.

O somatório dos percentuais de perdas de massa dos dois principais eventos térmicos $(97,46 \%)$ foi relativamente próximo ao percentual de conversão em ésteres metílicos, determinado por RMN $(89,1 \%)$ e, além disto, os valores de perda de massa apresentaram uma correlação positiva com os percentuais de ésteres metílicos insaturados $(70,66 \%)$ e saturados $(30,78 \%)$ observados na análise por CG (Tabela 1).

O terceiro evento térmico $\left(418,44-493,35{ }^{\circ} \mathrm{C}\right)$, com perda de massa de $1,61 \%$, deve ser referente aos resíduos de intermediários da reação, tais como, mono e diacilglicerois. Esta porcentagem encontra-se relativamente próxima ao teor obtido por CG $(1,17 \%)$, apresentado na Tabela 2.

\section{Parâmetros físico-químicos do biodiesel de garampara}

A maioria dos parâmetros físico-químicos determinados para o biodiesel de garampara (Tabela 2) estão dentro dos limites estabelecidos na Resolução 07/2008 da ANP, ${ }^{3}$ com exceção da estabilidade à oxidação e teor de glicerol total.

A estabilidade à oxidação foi de $2,97 \mathrm{~h}$, sendo superior ao relatado para o biodiesel de soja $0,16 \mathrm{~h}^{24}$ e $1,36 \mathrm{~h},{ }^{25}$ indicando a necessidade de correção com a adição de antioxidantes para atender às especificações (mínimo de 6 h). O teor de glicerol total $(1,1732 \%)$ é decorrente, em parte, da presença de monoacilglicerois, intermediários de menor reatividade, porém uma otimização da reação poderá corrigir este parâmetro. 
Tabela 2. Parâmetros físico-químicos do biodiesel de garampara

\begin{tabular}{|c|c|c|c|c|}
\hline Característica & Unidade & Método de ensaio & Resultado & Limite ANP ${ }^{6}$ \\
\hline Aspecto & - & - & LII & LII \\
\hline Viscosidade cinemática a $40^{\circ} \mathrm{C}$ & $\mathrm{mm}^{2} / \mathrm{s}$ & ASTM D 445 & 5,1332 & $3,0-6,0$ \\
\hline Ponto de fulgor, min & ${ }^{\circ} \mathrm{C}$ & ASTM D 93 & 156,0 & 100,0 \\
\hline Corrosividade ao $\mathrm{Cu}, 3 \mathrm{~h} 50^{\circ} \mathrm{C}$, máx. & - & ASTM D 130 & $1 \mathrm{a}$ & 1 \\
\hline Índice de acidez, máx. & $\mathrm{mg} \mathrm{KOH} / \mathrm{g}$ & IAL & 0,0206 & 0,50 \\
\hline Glicerol livre, máx. & $\%$ massa & ASTM D 6584 & 0,0012 & 0,02 \\
\hline Glicerol total, máx. & $\%$ massa & ASTM D 6584 & 1,1732 & 0,25 \\
\hline Monoacilglicerois & $\%$ massa & ASTM D 6584 & 1,1695 & Anotar \\
\hline Diacilglicerois & $\%$ massa & ASTM D 6584 & 0,0025 & Anotar \\
\hline Triacilglicerois & $\%$ massa & ASTM D 6584 & 0,0000 & Anotar \\
\hline Metanol, máx. & $\%$ massa & EN 41110 & 0,01 & 0,20 \\
\hline Estabilidade à oxidação $110^{\circ} \mathrm{C}$, min & $\mathrm{h}$ & EN 14112 & 2,97 & 6 \\
\hline
\end{tabular}

IAL: Instituto Adolfo Lutz. ${ }^{9}$ ANP: Agência Nacional de Petróleo, Gás Natural e Biocombustíveis. ${ }^{3}$ ASTM: American Society of Testing and Materials. EN: Européen Normalisation.

\section{Efeito da adição de antioxidantes ao biodiesel de garampara}

O biodiesel de garampara mostrou-se bastante sensível à oxidação, evidenciada pelo baixo tempo de indução determinado no Rancimat (2,97 h), isso pode ser atribuído ao alto teor de ácidos graxos insaturados (C18:1; C18:2; C20:1 e C22:1; 70,66\%) presentes na composição do óleo e, consequentemente, no biodiesel. Entretanto, este fato não desqualifica o biodiesel de garampara, pois se pode adicionar antioxidantes para aumentar a estabilidade e colocá-lo dentro da especificação (mínimo de $6 \mathrm{~h}$ ), como vem sendo feito com o biodiesel de soja, que também apresenta baixa sensibilidade à oxidação. Para tanto, realizaram-se testes utilizando dois antioxidantes sintéticos, tert-butil-hidroquinona (TBHQ) e butil-hidroxi-tolueno (BHT), e outro de origem natural, o líquido da casca da castanha de caju (LCC).

O TBHQ e o BHT (Figura 2) são antioxidantes primários que interrompem as reações oxidativas em cadeia, através da doação de hidrogênios das hidroxilas fenólicas aos radicais livres dos ésteres, transformando-se em radicais livres estabilizados por ressonância, evitando assim a iniciação e propagação da oxidação. ${ }^{24,26} \mathrm{O}$ efeito desses antioxidantes na estabilização do biodiesel de soja, palma e pinhão-manso já foi testado por diversos pesquisadores. ${ }^{24,27-30}$<smiles>Cc1cc(C(C)(C)C)c(O)c(C(C)(C)C)c1</smiles>

BHT<smiles>CC(C)(C)c1cc(O)ccc1O</smiles>

TBHQ

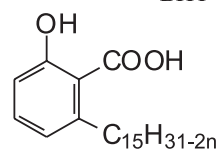

Ácido anacárdico

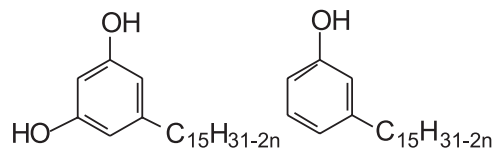

$$
\text { Cardol }
$$

Cardanol

$$
\mathrm{n}=0 ; \mathrm{n}=1 \Delta^{8} ; \mathrm{n}=2 \Delta^{8,11} ; \mathrm{n}=3 \Delta^{8,11,14}
$$

Figura 2. Estrutura química dos antioxidantes sintéticos (BHT e TBHQ) e dos principais constituintes do LCC

O LCC (Figura 2) é constituído por meta-alquilfenóis (ácido anacárdico, cardanol, cardol e traços de 2-metilcardol) que atuam como sequestradores de radicais livres e, algumas vezes, como quelantes de metais, nas etapas de iniciação e propagação do processo oxidativo. ${ }^{24} \mathrm{O}$ LCC pode ser utilizado como antioxidante de combustíveis e lubrificantes, sendo relatada na literatura a ocorrência de uma melhoria da estabilidade térmica do óleo e biodiesel de mamona, utilizando o LCC. ${ }^{31}$

A influência dos antioxidantes sintéticos sobre a estabilidade à oxidação do biodiesel metílico de garampara, utilizando diferentes concentrações, está mostrada na Figura 3. Observou-se um aumento do período de indução com o aumento da concentração do antioxidante adicionada ao biodiesel, sendo que o TBHQ apresentou melhores resultados do que o BHT, nas mesmas concentrações, em concordância com o relatado na literatura. ${ }^{28}$

A adição de 50 ppm de BHT ao biodiesel de garampara proporcionou uma elevação no período de indução, passando de 2,97 para $6,06 \mathrm{~h}$, enquanto que a mesma quantidade de TBHQ aumentou para 8,08 h. Este resultado mostrou-se semelhante ao relatado para o biodiesel do óleo de palma, com estabilidade à oxidação de 3,52 h, porém ao se adicionar BHT e TBHQ o período de indução aumenta para 6,42 e $8,85 \mathrm{~h}$, respectivamente. ${ }^{28}$

Na concentração de 100 ppm de ambos os antioxidantes observouse que os tempos de indução não apresentam diferença significativa, ao aplicar-se o teste ANOVA, a um nível de significância de 5\%, entretanto, a 500 ppm o TBHQ $(22,40 \mathrm{~h})$ apresenta eficácia superior ao BHT (14,43 h).

A adição de LCC (líquido da casca de castanha de caju) ao biodiesel de garampara melhorou a sua estabilidade à oxidação, observando-se também um aumento gradual no período de indução (Figura 3). O limite estabelecido pela Resolução $\mathrm{ANP}^{3}$ foi atingido na concentração de 2000 ppm, ou seja, 0,2\% de antioxidante, obtendose um valor de $6,02 \mathrm{~h}$, portanto, foi menos eficiente do que o BHT e TBHT, porém deve-se considerar que nesse estudo utilizou-se o LCC in natura, sem nenhum isolamento das substâncias antioxidantes presentes em sua composição.

Apesar do TBHQ e BHT serem mais efetivos do que o LCC, no aumento da estabilidade à oxidação do biodiesel, este último poderá ser economicamente viável, sobretudo nos países com grande produção de caju. Dessa forma, foram realizados testes com a utilização do LCC juntamente com o TBHQ. Para tanto, foi fixada a concentração do TBHQ em 25 ppm e efetuada a medida da estabilidade à oxidação com diferentes concentrações de LCC. Os resultados obtidos estão indicados na Figura 3. 

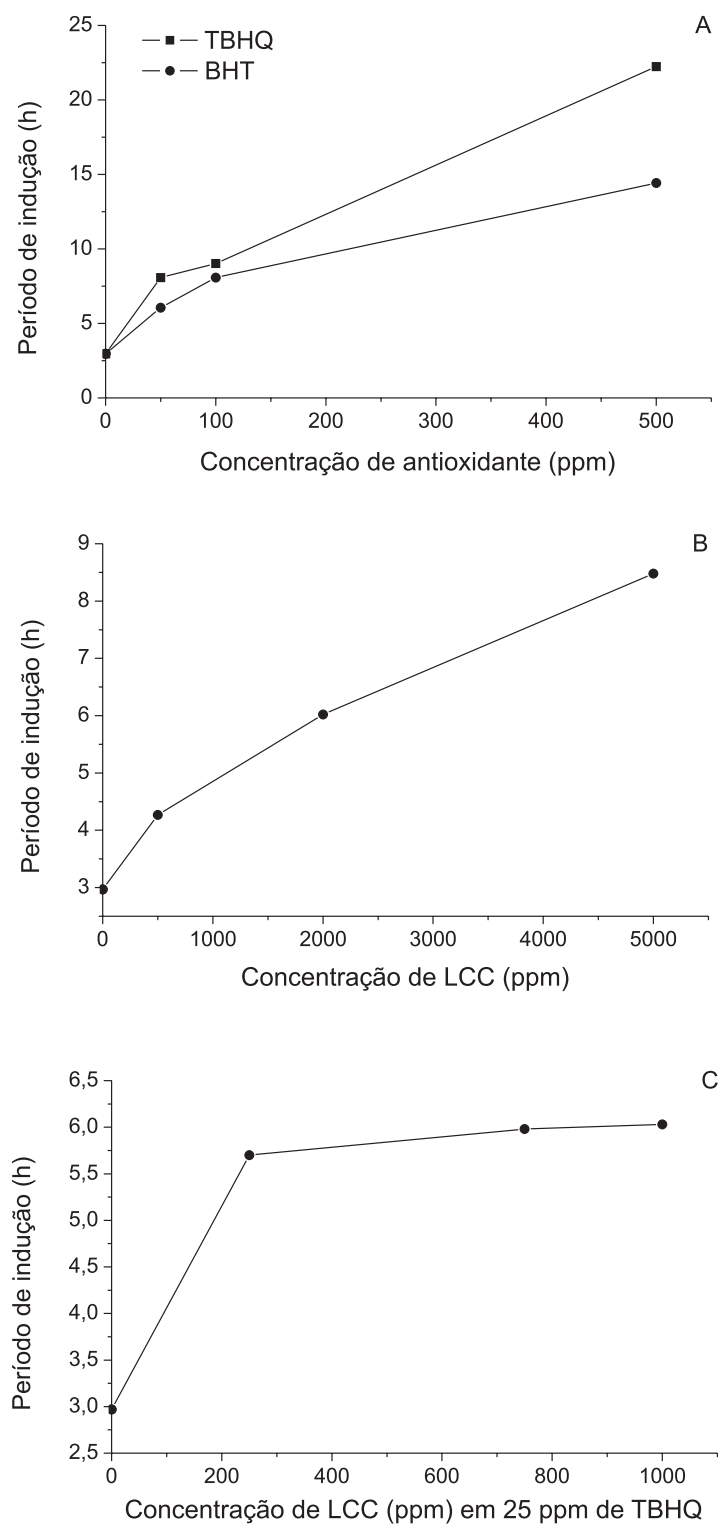

Figura 3. Efeito da adição de antioxidantes no aumento do período de indução à oxidação do biodiesel de garampara

Um aumento significativo do período de indução à oxidação foi observado ao se utilizar a mistura 25/250 ppm (TBHQ/LCC), com uma pequena melhoria gradual na estabilidade com o acréscimo de LCC. O limite de 6 h, estabelecido na Resolução da ANP, ${ }^{3}$ foi alcançado com 25/1000 ppm (TBHQ/LCC).

\section{CONCLUSÃO}

As amêndoas de Dipteryx lacunifera Ducke (garampara) apresentaram rendimento em óleo de $46,11 \pm 0,37 \%$, sendo o ácido oleico o constituinte principal dos triacilglicerois, determinado como éster metílico $(64,71 \pm 1,35)$.

As análises por $\mathrm{CCD}$ e RMN ${ }^{1} \mathrm{H}$ confirmaram a ocorrência da reação de transesterificação dos triacilglicerois do óleo, com uma taxa de conversão em ésteres metílicos de $89,1 \%$. As curvas TG/ DTG indicaram a ocorrência da reação de transesterificação pelo deslocamento do evento principal, observado na curva do óleo, para temperaturas menores, tendo em vista que o ponto de ebulição dos ésteres metílicos é inferior ao dos triacilglicerois.
O biodiesel metílico do óleo de garampara exibiu parâmetros dento das especificações da ANP, porém a estabilidade à oxidação foi inferior ao valor estabelecido; entretanto, a adição dos antioxidantes sintéticos TBHQ e BHT, bem como do líquido da casca de castanha de caju (LCC), ao biodiesel, proporcionou um aumento do período de indução à oxidação e consequente atendimento das especificações, sendo o LCC menos eficiente que os demais.

Os resultados obtidos apontam a oleaginosa garampara com potencial para produção de biodiesel, contudo mais estudos devem ser realizados, sobretudo agronômicos, para se determinar a sua viabilidade tecnológica.

\section{MATERIAL SUPLEMENTAR}

A Figura $1 \mathrm{~S}$ contendo os espectros na região do infravermelho do óleo e do biodiesel metílico de D. lacunifera está disponível gratuitamente em http://quimicanova.sbq.org.br, na forma de arquivo PDF.

\section{AGRADECIMENTOS}

Ao Laboratório da Brasil Ecodiesel Associado à Pesquisa e Desenvolvimento/Eusébio-CE pela disponibilização dos equipamentos necessários para a caracterização físico-química do biodiesel. Ao NuBBE/UNESP/Araraquara-SP pelos espectros de RMN. Ao LAPETROUFPI pelas análises de CG-EM e TG. Ao CNPq, CAPES e FINEP pelas bolsas de M. H. Chaves e F. D. S. Araújo e pelo apoio financeiro.

\section{REFERÊNCIAS}

1. Lima, J. R. O.; Silva, R. B.; Silva, C. C. M.; Santos, L. S. S.; Santos Jr., J. R.; Moura, E. M.; Moura, C. V. R.; Quim. Nova 2007, 30, 600.

2. Rinaldi, R.; Garcia, C.; Marciniuk, A. V. R.; Schuchardt, U.; Quim. Nova 2007, 30, 1364; Lobo, I. P.; Ferreira, S. L. C.; Cruz, R. S.; Quim. Nova 2009, 32, 1596.

3. http://www.anp.gov.br, acessada em Abril 2008 e Agosto 2010.

4. Ferrari, R. A.; Oliveira, V. S.; Scabio, A.; Quim. Nova 2005, 28, 19.

5. Pereira, R. G.; Oliveira, C. D.; Oliveira, J. L.; Oliveira, P. C. P.; Fellows, C. E.; Piamba, O. E.; Renewable Energy 2007, 32, 2453.

6. Guimarães, A. R. C.; Souza, V. A. B.; Vale, E. M.; Resumos do XX Congresso Brasileiro de Fruticultura e 54th Annual Meeting of the Interamerican Society for Tropical Horticulture, Vitória, Brasil, 2008.

7. Vieira Júnior, G. M.; Silva, H. R.; Bittencourt, T. C.; Chaves, M. H.; Quim. Nova 2007, 30, 1658.

8. Mendes, F. N. P.; Silveira, E. R.; Phytochemistry 1994, 35, 1499; Queiroga Neto, V.; Bora, P. S.; Diniz, Z. N.; Cavalheiro, J. M. O.; Queiroga, K. F.; Ciênc. Agrotec. 2009, 33, 1601.

9. Instituto Adolfo Lutz; Normas Analíticas do Instituto Adolfo Lutz. Métodos químicos e físicos para análise de alimentos, $4^{\mathrm{a}}$ ed., IMESP: São Paulo, 2005.

10. Moretto, E.; Fett, R.; Tecnologia de Óleos e Gorduras Vegetais na Indústria de Alimentos, Livraria Varela: São Paulo, 1998.

11. IUPAC; Standard Methods for Analysis of Oils, Fats and Derivatives, Blackwell Scientific Plubications, $7^{\text {th }}$ ed., 1987, IUPAC Method 2.301, Report of IUPAC Working Group WG 2/87.

12. Chaves, M. H.; Barbosa, A. S.; Moita Neto, J. M.; Aued-Pimentel, S.; Lago, J. H. G.; Quim. Nova 2004, 27, 404.

13. Aued-Pimentel, S.; Lago, J. H. G.; Chaves, M. H.; Kumagai, E. E.; J. Chromatogr., A 2004, 1054, 235.

14. Kumar, M. S.; Ramesh, A.; Nagalingam, B.; Biomass \& Bioenergy 2003, 25,309 .

15. Vicente, G.; Martínez, M.; Aracil, J.; Bioresour. Technol. 2006, 98, 1724.

16. Meher, L. C.; Sagar, D. V.; Naik, S. N.; Renew. Sustain. Energy Rev. 2006, 10, 248. 
17. Goodrum, J. W.; Biomass and Bioenergy 2002, 22, 205.

18. Goodrum, J. W.; Geller, D. P.; Bioresour. Technol. 2002, 84, 75.

19. Goodrum, J. W.; Geller, D. P.; Lee, S. A.; Thermochim. Acta 1998, 311, 71.

20. Antoniassi, R.; Boletim do Centro de Pesquisa e Processamento de Alimentos 2001, 19, 353.

21. Mello, F. O. T.; Paulillo, L. F.; Vian, C. E. F.; Informações Econômicas 2007, 37, 28.

22. Foidl, N.; Foidl, G.; Sanchez, M.; Mittelbach, M.; Hackel, S.; Bioresour. Technol. 1996, 58, 77.

23. Geris, R.; Santos, N. A. C.; Amaral, B. A.; Maia, I. S.; Castro, V. D.; Carvalho, J. R. M.; Quim. Nova 2007, 30, 1365.

24. Domingos, A. K.; Saad, E. B.; Vechiatto, W. W. D.; Wilhelm, H. M.; Ramos, L. P.; J. Braz. Chem. Soc. 2007, 18, 416.
25. Ryu, K.; Bioresour. Technol. 2010, 101, S78.

26. Ramalho, V. C.; Jorge, N.; Quim. Nova 2006, 29, 755.

27. Dunn, R. O.; Fuel Process. Technol. 2005, 86, 1017.

28. Liang, Y. C.; May, C. Y.; Foon, C. S.; Ngan, M. A.; Hock, C. C.; Basiron, Y.; Fuel 2006, 85, 867

29. Loh, S. L.; Chew, S. M.; Choo, Y. M.; J. Am. Oil Chem. Soc. 2006, 83, 947.

30. Sarin, R.; Sharma, M.; Sinharay, S.; Malhotra, R. K.; Fuel 2007, 86, 1365.

31. Silva, M. C. D.; Conceição, M. M.; Fernandes Jr., V. J.; Santos, I. M. G.; Souza, A. G.; Resumos do $1^{\circ}$ Congresso da Rede Brasileira de Tecnologia de Biodiesel, Brasília, Brasil, 2006. 


\section{BIODIESEL METÍLICO DE Dipteryx lacunifera: PREPARAÇÃO, CARACTERIZAÇÃO E EFEITO DE} ANTIOXIDANTES NA ESTABILIDADE À OXIDAÇÃO

Francisca Diana da Silva Araújo, Carla Verônica Rodarte de Moura e Mariana H. Chaves*

Departamento de Química, Universidade Federal do Piauí, Campus Ministro Petrônio Portela, 64049-550 Teresina - PI, Brasil

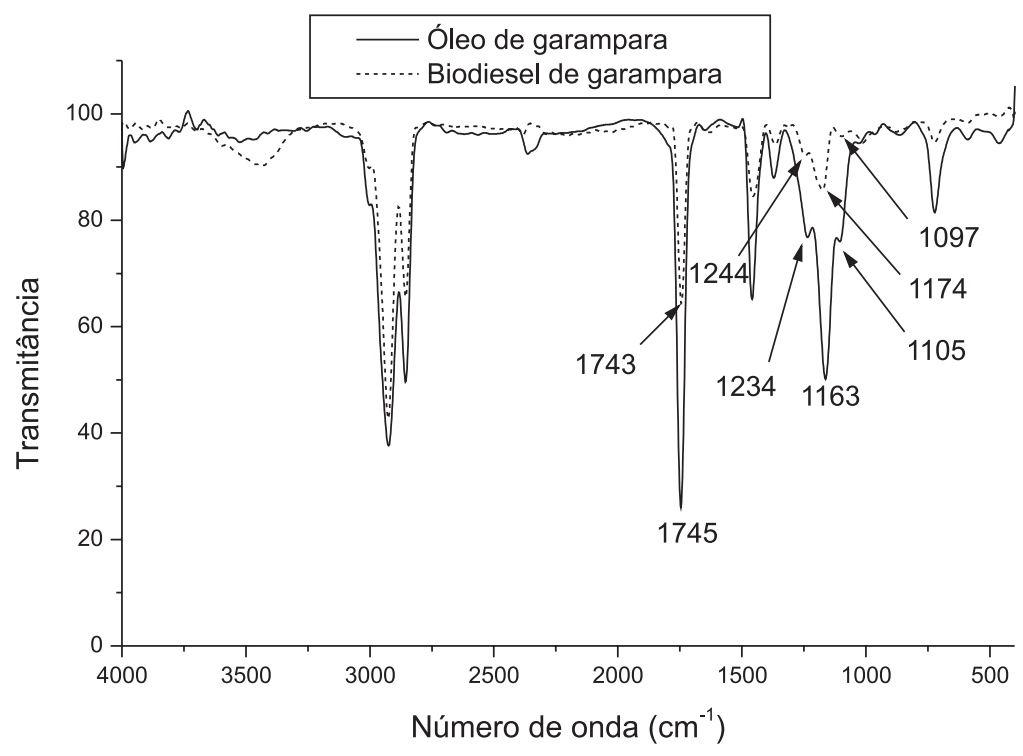

Figura 1S. Espectros de IV do óleo e biodiesel metílico de Dipteryx lacunifera 